\title{
Carrier-carrier entanglement and transport resonances in semiconductor quantum dots
}

\author{
Fabrizio Buscemi ${ }^{1,2, *}$ Paolo Bordone, ${ }^{1,2}$ and Andrea Bertoni ${ }^{1}$ \\ ${ }^{1}$ S3 Research Center, CNR-INFM, Via Campi 213/A, I-Modena 41100, Italy \\ ${ }^{2}$ Dipartimento di Fisica, Università di Modena e Reggio Emilia, 41100, Modena, Italy \\ (Received 14 June 2007; revised manuscript received 21 August 2007; published 16 November 2007)
}

\begin{abstract}
We study theoretically the entanglement created in a scattering between an electron, incoming from a source lead, and another electron bound in the ground state of a quantum dot, connected to two leads. We analyze the role played by the different kinds of resonances in the transmission spectra and by the number of scattering channels, into the amount of quantum correlations between the two identical carriers. It is shown that the entanglement between their energy states is not sensitive to the presence of Breit-Wigner resonances, while it presents a peculiar behavior in correspondence to Fano peaks: two close maxima separated by a minimum for a two-channel scattering and a single maximum for a multichannel scattering. Such a behavior is ascribed to the different mechanisms characterizing the two types of resonances. Our results suggest that the production and detection of entanglement in quantum dot structures may be controlled by the manipulation of Fano resonances through external fields.
\end{abstract}

DOI: $10.1103 /$ PhysRevB.76.195317

PACS number(s): 73.63.-b, 03.67.Mn, 03.65.Ud

\section{INTRODUCTION}

Quantum entanglement, as one of the most spectacular features of quantum mechanics contrasting with classical physics, ${ }^{1}$ has been widely investigated in the last decades, mainly because it is recognized as a crucial resource for quantum information processing and quantum communication. ${ }^{2}$ It is therefore a problem of great interest to find physical systems where the entanglement can be produced, manipulated, and detected. Recently, there have been different proposals to produce entangled states, such as those based on atomic systems, ${ }^{3}$ quantum electrodynamics cavities, ${ }^{4}$ and solid state devices.${ }^{5-10}$ Among the systems proposed for the realization of quantum information processing devices, those constructed with semiconductor quantum dots (QDs) are extremely promising due mainly to the controllability of their quantum state. ${ }^{11-13}$ Indeed, semiconductor QDs possess many desirable features: an atomiclike structure that can be fully controlled by an external electrostatical potential, a tunable coupling to source and drain leads, which makes the integration with other microelectronics devices feasible, and the scalability, which seems to promise sophisticated engineering of the multi-QD structures.

QDs represent also an ideal laboratory to compare exact numerical simulations of the quantum transport phenomena with experiments, since the number of degrees of freedom involved is often small and the discreteness of QD states highly reduces the computational burden needed. Various effects such as the conductance quantization, ${ }^{14}$ the Coulomb blockade due to the electron repulsion, ${ }^{15}$ the interplay between resonances and the charging in QD structures ${ }^{16,17}$ strongly affect the transport properties. Another peculiar feature of the electron transmission through QDs is the partial retention of quantum coherence, ${ }^{18}$ whose measurement, by experimental setups exploiting quantum interference (e.g., with a QD embedded in an Aharonov-Bohm interferometer), may yield information about transport phenomena, not readily available from conductance measures. ${ }^{19-21}$

In the frame of quantum transport in semiconductors, theoretical and experimental investigations have revealed other mechanisms governing the electronic transmission through QD structures. In particular, it has been observed that two kinds of resonances can be present in conductance spectra, known as Fano and Breit-Wigner resonances. ${ }^{22-26}$ The formers are present when two transmission channels, a resonant one and a nonresonant one, interfere. ${ }^{27}$ Moreover, they exhibit typical asymmetrical line shapes, with the transmitted phase increasing by $\pi$ on the resonance peak and then dropping abruptly. The latter show a symmetric line shape ${ }^{28}$ and are observed when only one channel is involved in the scattering process.

In this paper, we address the problem of entanglement generation in a two-particle scattering in a QD structure. We analyze, in particular, the role played by some mechanisms of charge transport in the appearance of quantum correlations. To this aim, we consider a scattering event in a onedimensional (1D) double-barrier resonant tunneling device (that mimics the confining potential of the QD), with an electron incoming from one lead and another electron bound in the ground state of the QD. The two particles feel the confining potential inside the device and interact through the Coulomb repulsion. Indeed, carrier-carrier entanglement has been recently investigated in various QD structures ${ }^{8,10,29-33}$ where different scattering setups are considered for the generation of two-electron entangled states. In the present work, we adopt a time-independent few-particle approach that, although computationally demanding, can be solved numerically to obtain the exact modulus and phase of the transmission coefficient (TC) of an electron crossing the charged QD. This gives us the possibility to quantify the quantum correlation between the energy states of the scattered electron and the bound one and to expose its connection with the resonances exhibited by the TCs of the various scattering channels. Unlike previous works, ${ }^{8,33}$ the few-particle approach we use in this paper allows us to study the relation between the different kinds of resonances, or the number of energy levels available, with the entanglement formation. Even if the dynamics of carriers has been considered as $1 \mathrm{D}$, we can assume that the results obtained describe a general behavior concerning also quantum transport in two-dimensional (2D) and 

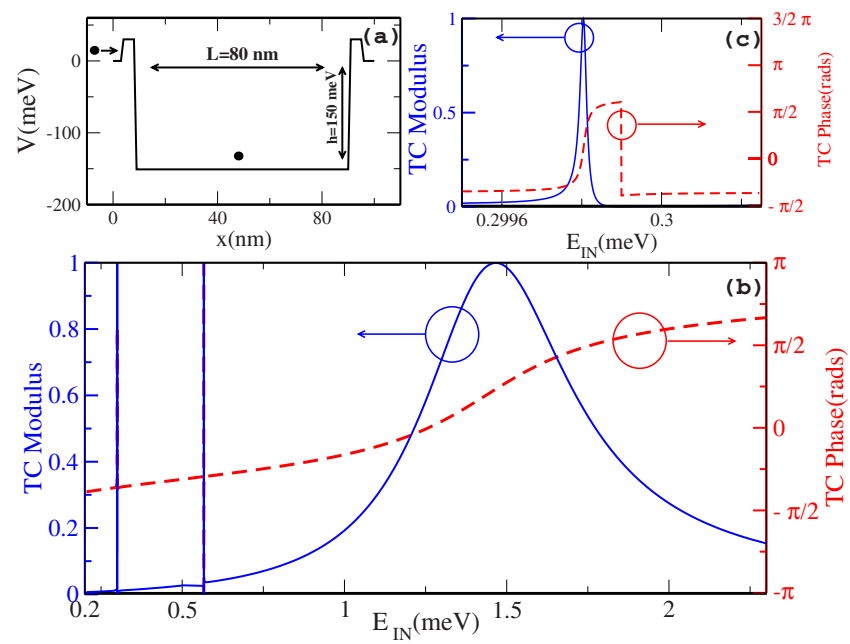

FIG. 1. (Color online) (b) TC as a function of the initial energy of the incoming electron $E_{I N}$. The solid line represents the modulus of the TC and refers to the left ordinate axis; the dashed line represents the phase of TC and refers to the right axis. (a) Profile of the potential $V(x)$ in the scattering region: the potential well is $150 \mathrm{meV}$ deep and $80 \mathrm{~nm}$ wide and is connected to the leads through two $10 \mathrm{~nm}$ barriers of $30 \mathrm{meV}$. (c) Zoom of the TC modulus and phase for $E_{I N} \simeq 0.3 \mathrm{meV}$ where a Fano resonance occurs.

three-dimensional physical systems, with the appearance of quantum correlations being closely related to the nature of transport resonances, whose underlying mechanisms are independent of the dimensionality of the system.

The paper is organized as follow. In Sec. II, we describe the physical model and the numerical approach adopted to calculate the two-particle scattering state and the quantum correlations in terms of the von Neumann entropy. In Sec. III, we present the numerical results obtained for the entanglement in the case of two different kinds of processes, namely, two- and multichannel scattering. ${ }^{43}$ Finally, in Sec. IV, we comment on the results, draw final remarks, and point out issues that require further research.

\section{PHYSICAL MODEL AND THE NUMERICAL APPROACH}

Our aim is to evaluate the entanglement between the energy states of two electrons, interacting via the Coulomb potential, one bound in a QD and the other passing through it. Here, we summarize the physical model adopted for the open QD and the numerical approach used to evaluate the two-particle scattering state and the entanglement.

We consider a quasi-1D double-barrier resonant tunneling device such as, for example, the ones formed by material modulation in a ultrathin cylindrical nanowire. ${ }^{34}$ The transversal dimensions of the structure are small compared to the other length scales so that a single transversal subband is accessible to the carriers and the effective dynamics can be considered 1D. ${ }^{35}$ Two small potential barriers separate the QD region from the two contacts, as depicted in Fig. 1(a). The $N$ bound states and energies of the QD will be indicated as $\chi_{n}$ and $E_{n}$ (with $n=0,1, \ldots, N$ in order of increasing energy), respectively.
A single electron is in the QD ground state $\chi_{0}$, whereas a second electron is incoming from the left lead with energy $E_{I N}$, and it is scattered by the structure potential $V_{s}$ and by the Coulomb interaction with the bound particle. The potential $V_{s}$ is supposed to be constant outside the region of interest of length $\Delta$ and, without loss of generality, it is taken to be zero in both left and right contacts. We will consider only cases in which the energy of the incoming electron is not sufficient to ionize the $\mathrm{QD}$, i.e., $\left(E_{I N}+E_{0}\right)<0$. This means that when an electron leaves the scattering region, either reflected or transmitted, the other one is in a bound state of the QD. Furthermore, we stress that in our system, the charging energy of the QD is much larger than the spacing between the singleparticle energy levels. For this reason, our system operates always in the two-particle regime. We do not include other carriers in our calculations.

The two-particle Hamiltonian reads

$$
\begin{aligned}
\mathbf{H}\left(x_{1}, x_{2}\right)= & -\frac{\hbar^{2}}{2 m^{*}} \nabla_{1}^{2}-\frac{\hbar^{2}}{2 m^{*}} \nabla_{2}^{2}+V_{s}\left(x_{1}\right)+V_{s}\left(x_{2}\right) \\
& +\frac{e^{2}}{4 \pi \epsilon \sqrt{\left(x_{1}-x_{2}\right)^{2}+d^{2}}},
\end{aligned}
$$

where $m^{*}$ and $\epsilon$ are the electron effective mass and the dielectric constant of the material, respectively. In particular, the calculations presented in this paper have been performed using GaAs material parameters. The Coulomb term includes the thickness, a cutoff term $d$ that can be assumed to correspond roughly to the lateral dimension of the confinement. ${ }^{35}$ The spin degree of freedom does not enter into our calculation since we neglect spin-orbit coupling. ${ }^{44}$. As a consequence of the fermionic nature of the system we impose antisymmetrization constraints under particle exchange to the wave function $\psi\left(x_{1}, x_{2}\right)$. This is done by adopting antisymmetric boundary conditions as briefly described in the following and detailed in Ref. 25.

The two-particle scattering state is obtained by solving the time-independent open-boundary Schrödinger equation $\mathbf{H} \psi$ $=E \psi$ in the $2 \mathrm{D}$ domain of interest. To this aim, we have used a numerical approach based on a generalization of the widely used quantum transmitting boundary method. ${ }^{36}$ It allows us to include proper open-boundary conditions and simulate the scattering of one electron by a charge confined in a QD. ${ }^{25} \mathrm{In}$ particular, here we need four boundary conditions, one for each edge of the square domain. Since we have to impose exchange symmetry to $\psi\left(x_{1}, x_{2}\right)$, they are equal in couples, apart from the sign. In fact, the form of the wave function when particle 1 is in the left lead $\left(x_{1}<0\right)$ is

$$
\begin{aligned}
\left.\psi\left(x_{1}, x_{2}\right)\right|_{\left(x_{1}<0\right)}= & \chi_{0}\left(x_{2}\right) e^{i k_{0} x_{1}} \\
& +\sum_{n=0}^{M} b_{n} \chi_{n}\left(x_{2}\right) e^{-i k_{n} x_{1}} \sum_{n=M+1}^{\infty} b_{n} \chi_{n}\left(x_{2}\right) e^{k_{n} x_{1}},
\end{aligned}
$$

and when it is particle 2, the one in the left lead, the boundary condition is $\left.\psi\left(x_{1}, x_{2}\right)\right|_{\left(x_{2}<0\right)}=-\left.\psi\left(x_{2}, x_{1}\right)\right|_{\left(x_{2}<0\right)}$. For the other two boundaries, namely, the conditions $\left(x_{1}>\Delta\right)$ and $\left(x_{2}>\Delta\right)$, it holds that 


$$
\left.\psi\left(x_{1}, x_{2}\right)\right|_{\left(x_{1}>\Delta\right)}=\sum_{n=0}^{M} c_{n} \chi_{n}\left(x_{2}\right) e^{i k_{n} x_{1}}+\sum_{n=M+1}^{\infty} c_{n} \chi_{n}\left(x_{2}\right) e^{-k_{n} x_{1}}
$$

and $\left.\psi\left(x_{1}, x_{2}\right)\right|_{\left(x_{2}>\Delta\right)}=-\left.\psi\left(x_{2}, x_{1}\right)\right|_{\left(x_{2}>\Delta\right)}$, respectively.

Let us now describe the above expressions. We first define $T_{n}=E_{I N}+E_{0}-E_{n}$ as the energy of an electron freely propagating in the lead when the other one is bound in $\chi_{n}$ (a consequence of energy conservation), and $k_{n}=\sqrt{2 m^{*}\left|T_{n}\right|} / \hbar$. The right hand side of Eq. (2) (for the left boundary) is the sum of three terms. The first one represents an electron incoming as a plane wave with energy $E_{I N}=\hbar^{2} k_{0}^{2} / 2 m^{*}$, while the other electron is in the QD ground state $\chi_{0}$. The second term accounts for all the energy-allowed possibilities with one electron bound in the $\chi_{n}$ state, and the other one reflected in the left lead, with wave vector $k_{n}$. $M$ is the number of states for which $T_{n}$ is positive. The third term accounts for the cases $T_{n}<0$, representing the electron in the lead as an evanescent wave. The right hand side of Eq. (3) (for the right boundary) has only two terms, since the probability amplitude of a carrier incoming from the right lead is zero in our system. The first term represents the $M$ energy-allowed possibilities of an electron transmitted and freely propagating in the right lead and the second term includes the outgoing-particle evanescent waves, as in Eq. (2). The reflection and transmission amplitudes in the various energy levels, $b_{n}$ and $c_{n}$, respectively, are unknown and are obtained by solving the Schrödinger equation with $\mathbf{H}\left(x_{1}, x_{2}\right)$ given by Eq. (1) and with the two-particle energy $E=E_{I N}+E_{0}$, imposing the two boundary conditions of Eqs. (2) and (3).

In order to describe the mechanisms characterizing the charge transport through a QD, and the kind of resonances showing up in transmission spectra, we report in Fig. 1 the modulus and phase of the TC as a function of the initial energy of the incoming electron, for a given configuration of the potential sketched in panel (a). In particular, we consider a width $L$ of the potential well of $80 \mathrm{~nm}$ with a depth $h$ of $150 \mathrm{meV}$. In this case, we have a single-channel scattering, i.e., the scattered particle can have a single energy, while the bound one is always left in the ground state of the QD well. When $E_{I N}$ is around $1.5 \mathrm{meV}$, the TC shows a Breit-Wigner resonance, as shown in panel (b). ${ }^{28}$ As it is well known, such kinds of resonances stem from the coupling of a quasibound state to the scattering states in the leads and present, a Lorentzian line shape whose amplitude $c_{0}$ is described by the expression $c_{0}=C(i \Gamma / 2) /\left(E_{I N}-E_{q b}+i \Gamma / 2\right)$, where $C$ is a complex constant and $\Gamma$ the width of the resonance, inversely proportional to the lifetime of the quasibound state with energy $E_{q b}$. In particular, as we can see from Fig. 1(b), the TC modulus (solid line) goes to 1 with a symmetric Lorentzian peak around the energy resonance, while the transmission phase (dashed line) smoothly changes by $\pi$. In addition to the above resonance, two extremely sharp resonances, for $E_{I N}$ around 0.3 and $0.6 \mathrm{meV}$ are present. They are the socalled Fano resonances (with asymmetric line shape), which can be ascribed to an effect brought about by electronelectron correlation. ${ }^{27}$ In fact, they originate from the interference of two alternative scattering mechanisms. ${ }^{23,25}$ In the first one, the injected particle gives part of its energy to the bound one, thus creating an autoionizing ${ }^{27}$ state where the two electrons are in two excited bound states of the QD; then one of the particles decays to the ground state and releases back its energy to the second one that is emitted. In the second mechanism, the electron entering the QD is scattered by the sum of the QD confining potential and the Coulomb repulsion with the bound particle. In both cases, Coulomb interaction plays an active role, with a remarkable difference: While in the second mechanism it represents simply a modification of the resonant energy, the first mechanism would not be possible without it. In fact, when the Coulomb potential is switched off, Fano resonances disappear, since only one scattering mechanism is now possible.

In order to give a better insight into their properties, we have reported in the top panel (c) a zoom of the modulus and phase of the TC at an initial energy of the incoming electron $E_{I N}$ around $0.3 \mathrm{meV}$. Here, the modulus reaches 1 and then goes to zero, showing the typical asymmetric Fano line shape. The transmission phase increases smoothly by $\pi$ on the resonance peak but shows an abrupt drop of $\pi$ in correspondence to the zero of the transmission probability. ${ }^{37} \mathrm{We}$ stress that the curves in Fig. 1 correspond to a single-channel two-particle scattering, with the energy of the transmitted electron fixed by the boundary conditions. As a consequence, no entanglement is generated between the two electrons here. The more interesting cases of two- and multichannel scattering will be presented in the following section.

In the last part of this section, we describe the procedure we adopt to evaluate the entanglement. In fact, as a consequence of the scattering, quantum correlations appear between the energy levels $E_{n}$ of the electron bound in the potential well and the energies $T_{n}$ of the scattered electron allowed by energy conservation. Such an entanglement may be evaluated from the transmitted component of the twoparticle wave function in the right lead, given by Eq. (3). Obviously only the traveling components, with $n \leqslant M$ in the right hand side of Eq. (3), must be considered, since they are the only ones giving a nonvanishing contribution to the current and also the only ones that could be revealed by measuring the energy of the electron propagating in the right lead. On the other hand, the evanescent component of the wave function plays no direct role in the appearance of detectable quantum correlations. In this case, a good entanglement measure is given by the von Neumann entropy of the reduced density matrix $\rho_{\text {red }}$, obtained by tracing the twoparticle density matrix $\rho=\left|\Psi^{t r}\right\rangle\left\langle\Psi^{t r}\right| /\left\langle\Psi^{t r} \mid \Psi^{t r}\right\rangle$ over the degrees of freedom of one of the particles, ${ }^{38}$ where $\psi\left(x_{1}, x_{2}\right)^{t r}$ $=\sum_{n=0}^{M} c_{n} \chi_{n}\left(x_{2}\right) e^{i k_{n} x_{1}}$.

$\rho_{\text {red }}$ will be a $(M+1) \times(M+1)$ diagonal matrix defined as

$$
\rho_{\text {red }}=\operatorname{diag}\left[\left|\widetilde{c}_{0}\right|^{2}, \ldots,\left|\widetilde{c}_{n}\right|^{2}, \ldots,\left|\widetilde{c}_{M}\right|^{2}\right]
$$

with

$$
\tilde{c}_{n}=\frac{c_{n}}{\sqrt{\sum_{n=0}^{M}\left|c_{n}\right|^{2}}}
$$

Thus, the entanglement can be expressed by means of the von Neumann entropy as 


$$
\varepsilon=-\operatorname{Tr}\left[\rho_{\text {red }} \ln \rho_{\text {red }}\right]=-\sum_{n=0}^{M}\left|\widetilde{c}_{n}\right|^{2} \ln \left|\widetilde{c}_{n}\right|^{2} .
$$

$\varepsilon$ is bound in the interval $[0, \ln (M+1)]$, with $\varepsilon=0$ indicating no entanglement and $\varepsilon=\ln (M+1)$ indicating a maximally entangled state. As shown in previous works, the von Neumann entropy allows us to quantify the quantum correlations induced by the Coulomb interaction. In fact, if noninteracting electrons are considered, only one transmission channel is active, and, as a consequence, the von Neumann entropy results to be zero. We stress that, unlike other works estimating the quantum correlation in QDs, ${ }^{8,33}$ we are not considering the transmitted and reflected components of the scattering wave function as two different states that can be entangled with the QD. We estimate the entanglement between the dot and the transmitted electron. In fact, in our approach, the measure of the entanglement created in the system, evaluated by means of Eq. (6), is not explicitly given in terms of the device transmission and reflection coefficients, but it is a function of the amplitude probabilities of finding the transmitted particle in one of the possible energy states. Furthermore, it is worth noting that, although we do not use explicitly the criteria developed to treat the entanglement of identical particles, ${ }^{5,39,40}$ the electrons in our system are fully indistinguishable. Nevertheless, since the energy of the incoming electron is not sufficient to ionize the QD, we may safely assume that the scattered particle is far enough from the one left in a bound state of the dot so that the overlap between their spatial probabilities is negligible. In other words, we use the spatial position of the electrons to "distinguish" them, while the entanglement is between their energy states, as suggested in Ref. 41.

\section{NUMERICAL RESULTS}

In this section, we analyze the entanglement formation in a two- or multichannel scattering and its relation to the resonances in the TCs. In particular, it is of interest to study how the entanglement depends on the kind of resonances, since the detection of the latter, usually feasible through a currentvoltage characteristic of the quantum device, can give information about the former. In our approach, we solve numerically the system for different potentials $V(x)$ (obtained by varying the depth $h$ and the width $L$ of the well) and for different initial energies of the incoming electron $E_{I N}$.

In panel (a) of Fig. 2, we report the entanglement $\varepsilon$ of the system as a function of the initial energy of the incoming electron and of the depth of the potential well, whose width is kept constant at $L=40 \mathrm{~nm}$. The entanglement presents extremely sharp peaks that, as we shall see, correspond to the resonances exhibited by the TCs of the various channels. From the 2D representation of the same data, displayed in Fig. 2(b), we observe that the maxima of the entanglement spread in the region corresponding to a three-channel scattering, which is separated by the dashed line from the one where the number of active channels is 2 . This suggests us that also the kind of process, i.e., two- or multichannel scattering, plays an important role into the entanglement forma-
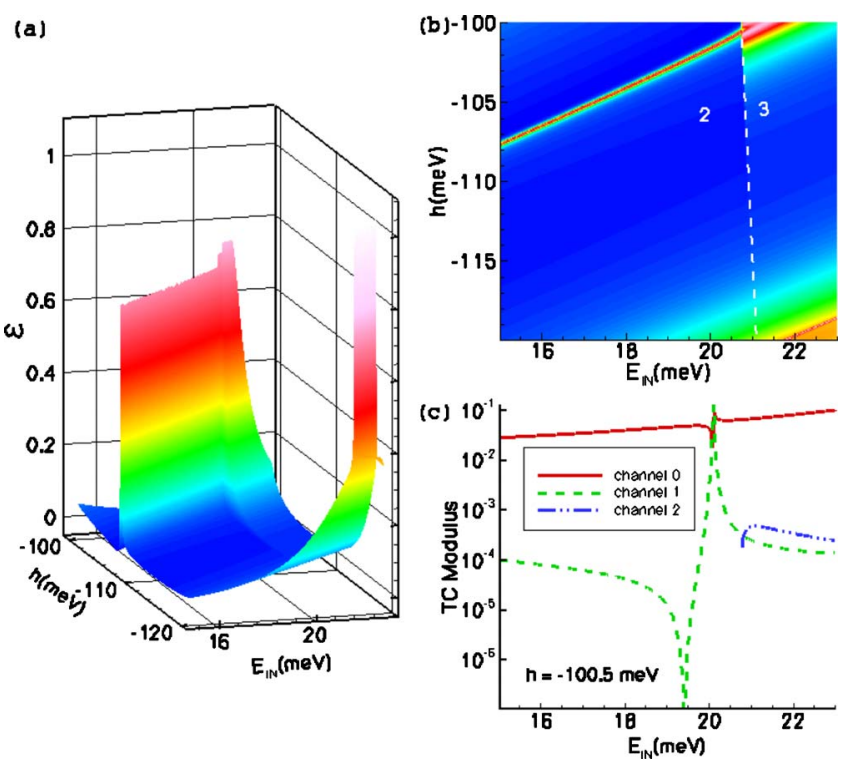

FIG. 2. (Color online) (a) Entanglement $\varepsilon$ as a function of the initial energy of the incoming electron $E_{I N}$ and of the depth of the potential well of $40 \mathrm{~nm}$. (b) 2D contour plot of the same data; the dashed line is a guide for the eyes and separates the zone corresponding to two-channel scattering (left) from the one corresponding to a three-channel scattering (right). (c) Modulus of TC of the three channels as a function of $E_{I N}$ with $h$ fixed at $-100.5 \mathrm{meV}$. Note that channel 2 is activated only at $E_{I N} \simeq 21 \mathrm{meV}$.

tion. The two cases will be better analyzed in the following sections.

\section{A. Two-channel scattering}

Here, we study the creation of the entanglement when the incident particle, as a consequence of the scattering with the particle bound in the QD, is transmitted with two possible energies, $T_{0}$ and $T_{1}$, and, correspondingly, the final QD energy can be $E_{0}$ and $E_{1}$.

In the top panel of Fig. 3, we report the entanglement as a function of the kinetic energy of the incoming particle for the potential sketched in the inset of the figure. In particular, we consider a width of the potential well of $30 \mathrm{~nm}$ and a depth of $110 \mathrm{meV}$. At low energies, the scattering does not lead to the appearance of quantum correlations between the two particles, since only a single channel is possible for the transmission. It is therefore possible to attribute a specific energy to each particle: $T_{0}$ for the scattered electron and $E_{0}$ for the bound one. When $E_{I N}$ reaches a threshold value of about $14 \mathrm{meV}$, a new channel comes into play as it can be seen from the bottom panel of Fig. 3. There, the dependence of the modulus of the TC is reported as a function of the initial energy of the incoming electron. In correspondence to the energy at which the second channel is activated, the entanglement shows a sharp increase.

By comparing the two panels in Fig. 3, it is clear that the resonances of the TCs play a key role in the entanglement formation. In particular, the behavior shown by the entanglement in correspondence to a Fano resonance is very different 

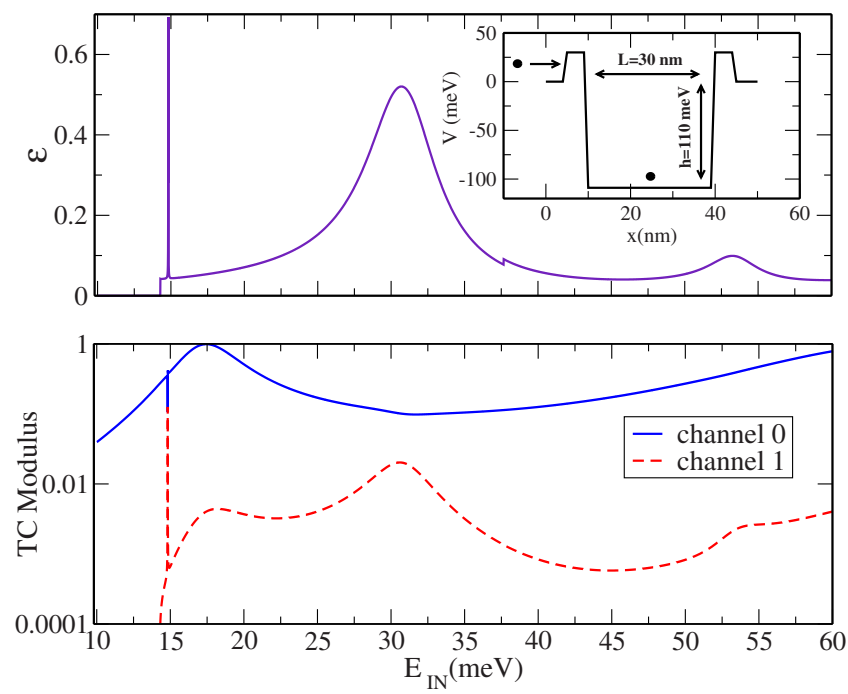

FIG. 3. (Color online) (Top panel) The entanglement $\varepsilon$ as a function of the initial energy of the incoming electron $E_{I N}$. The inset displays the potential $V(x)$ inside the scattering region; we consider a potential well $110 \mathrm{meV}$ deep and $30 \mathrm{~nm}$ wide. (Bottom panel) The modulus of the TC of the channels, in logarithmic scale, as a function of the initial kinetic energy of the incident electron: zeroth channel (solid line) and first channel (dashed line). The scale of the abscissa is the same in the two panels. Note that the sum of the moduli of the TCs is always $\leqslant 1$.

from the one exhibited at a Breit-Wigner resonance. When $E_{I N}$ is around $15 \mathrm{meV}$, a Fano resonance of the TC is observed for channel 0 . To better understand this phenomenon, we report in the top panel of Fig. 4 a zoom of the curves of modulus and phase (the latter was not shown in Fig. 3 for clarity) of the TCs of the two channels. It is worth noting that, unlike the case of the single-channel scattering, here the modulus of the TC attains small but nonzero values before the maximum, which, in turn, results to be significantly lower than 1 . The TC of channel 1 shows, in correspondence to the Fano resonance of channel 0, a Breit-Wigner resonance characterized by a phase change of about $\pi$. In the energy interval around $15 \mathrm{meV}$, the behavior of the quantum correlations, appearing in the systems as a consequence of the scattering, is peculiar (see the top panel of Fig. 4). In fact, when the modulus of the TC of channel 0 reaches its lowest value, the entanglement curve presents a minimum. Such a minimum is placed between two very close maxima, where the entanglement (evaluated, as usual, by means of the von Neumann entropy of the reduced density matrix) is equal to $\ln 2$. This value indicates the condition of maximal entanglement in a two-channel scattering. Such a condition is reached when $\left|c_{0}\right|^{2}$ and $\left|c_{1}\right|^{2}$, i.e., the probabilities that the scattering occurs through the channel 0 or 1 , respectively, are equal, and it implies that the lack of knowledge about the state in the one-particle subspace is maximum. We also report, in the top panel of the Fig. 4 (dashed line), the ratio of the two transmission probabilities: the entanglement is maximum when the scattering probabilities in the two channels are the same, as indicated by the horizontal dotted line drawn as a guide for the eyes. What we found here is in agreement with
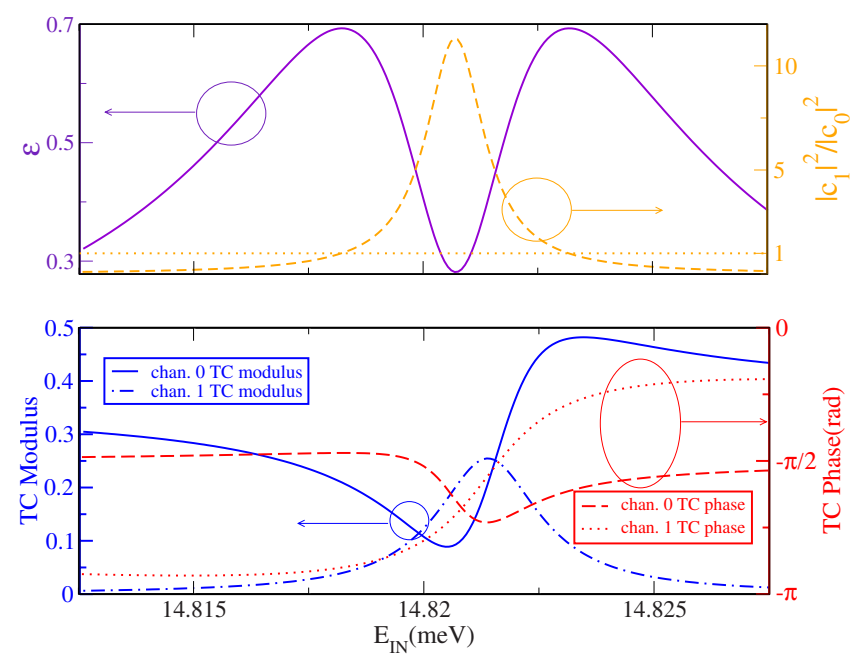

FIG. 4. (Color online) (Top panel) The entanglement $\varepsilon$ (solid line) and ratio between the probabilities of finding the scattered particle in the transmission channels $1,\left|c_{1}\right|^{2}$, and $0,\left|c_{0}\right|^{2}$ (dashed line), against the initial energy of the incoming electron $E_{I N}$, close to a resonant condition. Note that, while $\varepsilon$ can range from 0 (no entanglement) to $\ln 2$ (maximally entangled state), the bottom of the left scale is set to 0.25 to optimize the magnification effect in the figure. The horizontal dotted line is a guide for the eyes, indicating the point of equal scattering probabilities for the two channels, i.e., 1 in the left ordinates axis. (Bottom panel) TC of the two channels as a function of $E_{I N}$ around a Fano resonance. The solid and the dash-dotted lines represent the modulus of TC of channels 0 and 1 , respectively, and refer to the left ordinate axis; the dashed and the dotted lines represent the phase of the TC of channels 0 and 1, respectively, and refer to the right axis.

previous analyses on the two-electron entanglement production in two-electron systems for a two-channel scattering model, where both particles are injected in only one of two leads. ${ }^{8,33}$ In fact, also in those cases, the entanglement shows a maximum when the transmission probabilities for the two channels are identical, while it vanishes in correspondence to the single-particle resonances, where there is no uncertainty about the energy of the particle.

Figure 3 shows that the TC of channel 0 presents a BreitWigner resonance for $E_{I N} \simeq 17.5 \mathrm{meV}$. Even if the modulus of the TC becomes equal to 1 , the entanglement curve does not display maxima or minima. This is due to the fact that the Breit-Wigner resonance of the TC of channel 0 does not influence the TC of channel 1, which does not show, at the specific energy, resonances of any kind. Therefore, the scattering phenomena taking place in the energy interval around $17.5 \mathrm{meV}$ do not play a special role into entanglement formation of the two-particle system. Such a behavior is in agreement with the one observed in other works, showing that the maximal value of the conductance does not always correspond to the maximal entanglement. ${ }^{30}$

\section{B. Multichannel scattering}

Let us consider now the case of a multichannel scattering (the scattered particle can leave the QD with more than two 


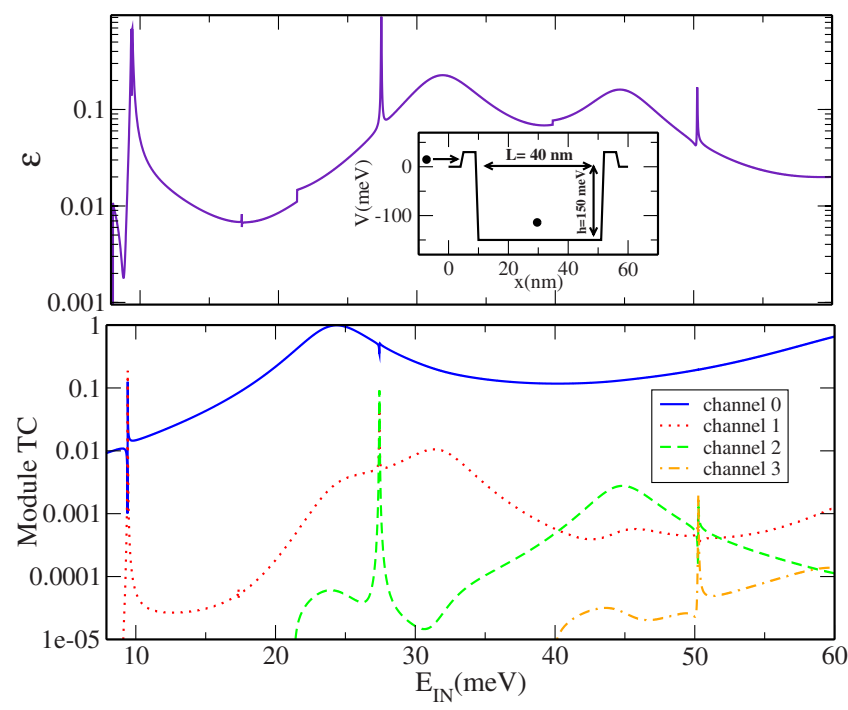

FIG. 5. (Color online) (Top panel) The entanglement $\varepsilon$ as a function of the initial kinetic energy of the incoming electron $E_{I N}$. The inset shows the profile of the potential $V(x)$ in the scattering region: the potential well is $150 \mathrm{meV}$ deep and $40 \mathrm{~nm}$ wide. (Bottom panel) Modulus of the TC of the four channels as a function of $E_{I N}$ : channels 0 (solid line), 1 (dotted line), 2 (dashed line), and 3 (dash-dotted line).

energies) and let us investigate the different roles played by the Fano and Breit-Wigner resonances in the entanglement formation.

Here, we consider a potential well $150 \mathrm{meV}$ deep and $40 \mathrm{~nm}$ wide, as reported in the inset of Fig. 5. For $E_{I N}$ around $21 \mathrm{meV}$, the scattering passes from a two-channel to a threechannel process, and this transition is characterized by a sharp increase of the entanglement. The same behavior is found at $E_{I N}=40 \mathrm{meV}$, where an additional transmission channel is switched on (see Fig. 5). This is in agreement with the results of the previous section and may be considered representative of a more general behavior, occurring whenever a new channel becomes effective in the scattering process.

The quantum correlations appearing in the system, as a consequence of a multichannel scattering, for the energy values around a Fano resonance show some differences with respect to the two-channel process. In Fig. 6, we report the curves of the entanglement and, in the insets, the modulus and phase of the TCs of the three channels $(0,1,2$ from top to bottom) against $E_{I N}$, in the energy region around $E_{I N}$ $=27.5 \mathrm{meV}$, where a Fano resonance occurs for channel 0. In fact, we observe, from the uppermost inset, that also in this case the modulus of the TC has a local minimum before reaching the maximum. The type of resonances of channels 1 and 2 (middle and bottom insets) cannot be clearly identified by the module of their transmission coefficients, whose peaks are almost symmetric. However, it is clear for the transmission phases that channel 1 exhibits a Fano resonance, with the phase essentially unchanged through the peak, while channel 2 shows a Breit-Wigner resonance, with a global phase variation of $\pi$. We stress that, unlike the twochannel scattering case, here the entanglement does not show

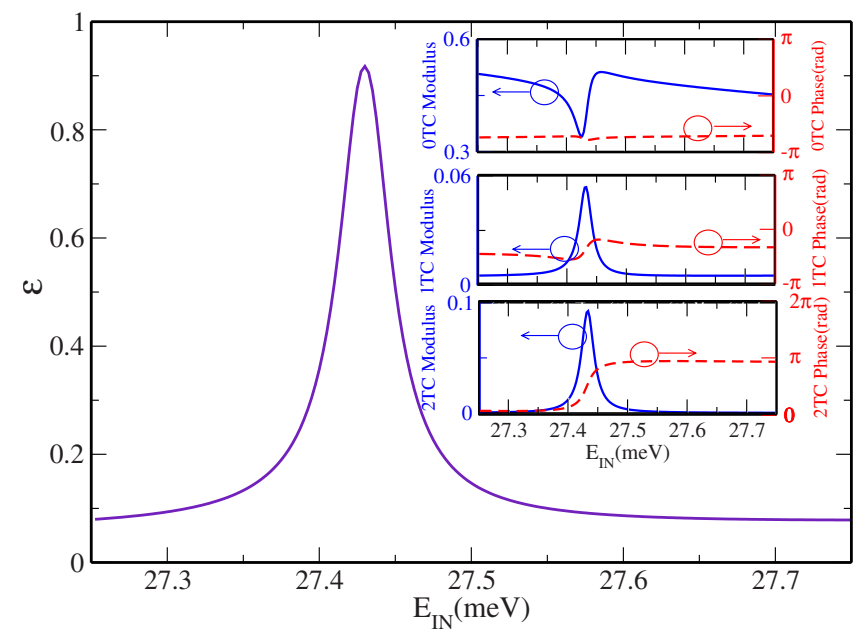

FIG. 6. (Color online) The entanglement $\varepsilon$ as a function of $E_{I N}$ around a Fano resonance of the TC of channel 0 in a three-channel scattering. The insets display the modulus (solid line, left axis) and phase (dashed line, right axis) of TC of three channels $(0,1,2$ from top to bottom) in the same energy range of the main graph.

a minimum. Such a behavior can be ascribed to the fact that, when the modulus of the TC of channel 0 is small, the TCs of the other two channels attain values comparable to each other. This means that the probabilities of finding the scattered particles in those channels are almost equal, and there is still a lack of knowledge about the state of the one-particle subsystem. Furthermore, we note that here the entanglement presents a single maximum whose value exceeds $\ln 2$. Actually, the fact that the number of degrees of freedom is larger than 2 increases the uncertainty about the constituents of the system; in fact, Eq. (6) gives a maximum value for the amount of quantum correlations that is larger for a larger number of possible states. For example, a system of two qutrits is able to attain a larger value of $\varepsilon$ than a two-qubit system. ${ }^{45}$ The ability to tailor not only the degree of the entanglement but also the number of possible states of the two subsystems, by independently tuning $E_{I N}$ and the QD confining potential, could also have implications beyond the theoretical estimation of the entanglement. The behavior described above is repeated at $E_{I N} \simeq 50 \mathrm{meV}$, where two Fano resonances occur for the second and third channels in a fourchannel scattering (Fig. 5). It is worth noting that, in correspondence to a Breit-Wigner resonance in channel 0 for $E_{I N}=25 \mathrm{meV}$, no additional resonance occurs in the other channels and the entanglement does not present maxima or minima as it can be clearly seen from the upper panel of Fig. 5. Therefore, also in the case of multichannel processes, the Breit-Wigner resonance seems not to induce sharp variations of $\varepsilon$.

\section{CONCLUSIONS}

The controlled production and detection of entangled particles in the solid state environment represents an experimental challenge. In this spirit, various proposals for producing bipartite entangled fermionic systems have been advanced, 
on the basis of different physical mechanisms requiring a direct interaction between particles. ${ }^{5,7-10,33}$ In this paper, we have investigated the quantum correlations appearing, as a consequence of a Coulomb scattering, between two electrons having the same spin, in a system of physical interest, where the degree of the entanglement results to be controllable by a proper tuning of the carrier energy and of the QD potential. Such a system consists of a quasi-1D double-barrier resonant tunneling device, where an electron incoming from one lead is scattered by the potential structure and, via the Coulomb interaction, by another electron bound in the QD.

The numerical procedure used to solve the model is a generalization of the quantum transmitting boundary method. ${ }^{25,36}$ It permits us to obtain the reflection and transmission amplitudes of each scattering channel, for various configurations of the potential, as a function of the initial energy of the incoming electron. However, we stress that, unlike the approaches followed by Lopez et $a l^{33}$ and by Oliver et al., ${ }^{8}$ here we did not use the reflected component of the scattered electron wave function to evaluate the entanglement of the two-particle system, but we estimated the quantum correlations showing up between the QD eigenstates and the transmitted parts of the electron wave function. Furthermore, this procedure makes it possible to investigate the role played by the resonances of the transmission spectra into the entanglement. Although our numerical analysis has been performed by using the GaAs material parameters, they can be considered representative of a more general behavior.

Our simulations show that the entanglement depends on the kind of the resonance appearing in the transmission spectrum. A single Breit-Wigner resonance is found not to induce peculiar effects on quantum correlations. On the contrary, in correspondence to a Fano resonance of one of the TCs, not only the other channels TCs exhibit local maxima, but also the entanglement presents sharp peaks. Such a behavior can be related to the nature of the resonances themselves. In fact, a Breit-Wigner resonance is essentially a one-particle effect, showing up also in a single-electron scattering. On the other hand, a Fano resonance is, in our 1D system, a genuine multiparticle phenomenon, due to electron-electron correlation. In fact, if Coulomb interaction is switched off, Fano resonances disappear from the transmission spectrum. We note that in different systems, with higher dimensionality, Fano resonances can occur due to the interference of two singleparticle transmission channels, as, for example, in a twodimensional QD or in a two-path interferometer. Thus, the presence of an asymmetric Fano line shape is a true signature of particle-particle correlation only in 1D systems.

Furthermore, we showed that the appearance of quantum correlations in our system is also affected by the number of the transmission channels, i.e., the number of possible energy levels of the scattered particle (and, due to the energy conservation, of the bound particle). In fact, the entanglement shows a sharp increase whenever a new channel is turned on. Moreover, its behavior for energy values around a Fano resonance is found to depend on the kind of process: two- or multichannel scattering. For the two-channel case, the entanglement presents a minimum between two close maxima, which indicate the maximal uncertainty about the state of the system. In the multichannel case, a single maximum of the entanglement, with no minima, is observed. When the energy levels of the scattered and bound electrons are only 2, the minimum of the entanglement is found in correspondence to the local minimum of the TC of the Fano resonant channel. In this case, it maximizes the possibility to ascribe specific energy states to the subsystems. On the other hand, for a multichannel scattering, a minimum of TC of a Fano resonant channel does not imply a decrease of uncertainty about the subsystems, since the TCs of the other non-Fano resonant channels attain values comparable to each other.

Finally, the results of our paper suggest that the manipulation of Fano resonances and of the number of scattering channels may allow to significantly influence the degree of entanglement between the transmitted electrons and the QD. A promising development of the present work could be the study of the entanglement in the case of scattering of a single electron by a few charges confined in the QD, in connection with experimental results obtained for the coherent components of the transmitted current in the case of the multioccupancy of the dot. ${ }^{18,42}$ In the latter case, with three or more particles in the system, the spin degrees of freedom cannot be factorized and their inclusion in our approach, although quite feasible, results to be very challenging from the computational point of view. *buscemi.fabrizio@unimore.it

${ }^{1}$ A. Einstein, B. Podolsky, and N. Rosen, Phys. Rev. 47, 777 (1935).

${ }^{2}$ D. Bouwnmeester, A. Ekert, and A. Zeilinger, The Physics of Quantum Information (Springer-Verlag, Berlin, 2000).

${ }^{3}$ W. H. Chan and C. K. Law, Phys. Rev. A 74, 024301 (2006).

${ }^{4}$ R. Lo Franco, G. Compagno, A. Messina, and A. Napoli, Phys. Rev. A 72, 053806 (2005).

${ }^{5}$ F. Buscemi, P. Bordone, and A. Bertoni, Phys. Rev. A 73, 052312 (2006).

${ }^{6}$ A. Bertoni, P. Bordone, R. Brunetti, C. Jacoboni, and S. Reggiani, Phys. Rev. Lett. 84, 5912 (2000).

${ }^{7}$ P. Bordone, A. Bertoni, and C. Jacoboni, J. Comput. Electron. 3,
407 (2004).

${ }^{8}$ W. D. Oliver, F. Yamaguchi, and Y. Yamamoto, Phys. Rev. Lett. 88, 037901(R) (2002).

${ }^{9}$ A. Ramsak, J. Mravlje, R. Zitko, and J. Bonca, Phys. Rev. B 74, 241305(R) (2006)

${ }^{10}$ A. Ramsak, I. Sega, and J. H. Jefferson, Phys. Rev. A 74, 010304(R) (2006).

${ }^{11}$ D. Loss and D. P. Di Vincenzo, Phys. Rev. A 57, 120 (1998).

${ }^{12}$ A. Imamoglu, D. D. Awschalom, G. Burkard, D. P. DiVincenzo, D. Loss, M. Sherwin, and A. Small, Phys. Rev. Lett. 83, 4204 (1999).

${ }^{13}$ G. Bester and A. Zunger, Phys. Rev. B 72, 165334 (2005).

${ }^{14}$ B. J. van Wees, H. van Houten, C. W. J. Beenakker, J. G. Will- 
iamson, L. P. Kouwenhoven, D. van der Marel, and C. T. Foxon, Phys. Rev. Lett. 60, 848 (1988).

${ }^{15}$ U. Meirav, M. A. Kastner, and S. J. Wind, Phys. Rev. Lett. 65, 771 (1990).

${ }^{16}$ W. G. van der Wiel, S. De Franceschi, J. M. Elzerman, T. Fujisawa, S. Tarucha, and L. P. Kouwenhoven, Rev. Mod. Phys. 75, 1 (2003).

${ }^{17}$ B. Lassen and A. Wacker, Phys. Rev. B 76, 075316 (2007).

${ }^{18}$ H. Aikawa, K. Kobayashi, A. Sano, S. Katsumoto, and Y. Iye, Phys. Rev. Lett. 92, 176802 (2004).

${ }^{19}$ A. Yacoby, M. Heiblum, D. Mahalu, and H. Shtrikman, Phys. Rev. Lett. 74, 4047 (1995).

${ }^{20}$ M. Avinun-Kalish, M. Heiblum, O. Zarchin, D. Mahalu, and V. Umansky, Nature (London) 436, 529 (2005).

${ }^{21}$ I. Neder, M. Heiblum, D. Mahalu, and V. Umansky, Phys. Rev. Lett. 98, 036803 (2007).

${ }^{22}$ J. Gores, D. Goldhaber-Gordon, S. Heemeyer, M. A. Kastner, H. Shtrikman, D. Mahalu, and U. Meirav, Phys. Rev. B 62, 2188 (2000).

${ }^{23}$ C. Fuhner, U. F. Keyser, R. J. Haug, D. Reuter, and R. J. Haug, arXiv:cond-mat/0307590 (unpublished)

${ }^{24}$ M. L. Ladron de Guevara and P. A. Orellana, Phys. Rev. B 73, 205303 (2006).

${ }^{25}$ A. Bertoni and G. Goldoni, J. Comput. Electron. 73, 205303 (2006).

${ }^{26}$ J. U. Nockel and A. D. Stone, Phys. Rev. B 50, 17415 (1994).

${ }^{27}$ U. Fano, Phys. Rev. 124, 1866 (1961).

${ }^{28}$ G. Breit and E. Wigner, Phys. Rev. 49, 519 (1936).

${ }^{29}$ P. I. Tamboronea and H. Metiu, Europhys. Lett. 53, 776 (2001).

${ }^{30}$ A. Rycerz, Eur. Phys. J. B 52, 291 (2006).

${ }^{31}$ L. D. Contreras-Pulido and F. Rojas, J. Phys.: Condens. Matter 18, 9971 (2006)
${ }^{32}$ T. Ihn, C. Ellenberger, K. Ensslin, C. Yannouleas, U. Landman, D. C. Driscoll, and A. C. Gossard, Int. J. Mod. Phys. B 21, 1316 (2007).

${ }^{33}$ A. Lopez, O. Rendon, V. M. Villaba, and E. Medina, Phys. Rev. B 75, 033401 (2007).

${ }^{34}$ M. T. Bjork, C. Thelander, A. E. Hansen, L. E. Jensen, M. W. Larsson, L. R. Wallenberg, and L. G. Samuelson, Nano Lett. 4, 1621 (2004).

${ }^{35}$ M. M. Fogler, Phys. Rev. Lett. 94, 056405 (2005).

${ }^{36}$ C. S. Lent and D. J. Kirkner, J. Appl. Phys. 67, 6353 (1990).

${ }^{37}$ A. Bertoni and G. Goldoni, Phys. Rev. B 75, 235318 (2007).

${ }^{38}$ A. Peres, Quantum Theory: Concepts and Methods (Kluwer, Dordrecht, 1995).

${ }^{39}$ J. Schliemann, J. I. Cirac, M. Kus, M. Lewenstein, and D. Loss, Phys. Rev. A 64, 022303 (2001).

${ }^{40}$ F. Buscemi, P. Bordone, and A. Bertoni, Phys. Rev. A 75, 032301 (2007).

${ }^{41}$ K. Eckert, J. Schliemann, D. Bruss, and M. Lewenstein, Ann. Phys. (N.Y.) 299, 88 (2002).

${ }^{42}$ J. Konig and Y. Gefen, Phys. Rev. Lett. 86, 3855 (2001).

${ }^{43}$ Here and in the following, we indicate, for brevity, as multichannel scattering a process with more than two channels, as detailed in Sec. III.

${ }^{44}$ In fact, we assume that the two particles are identical. This is justified if the two electrons are considered spinless or if they have the same spin. The only possible alternative, i.e., the two electrons having opposite spin, corresponds to the simpler case of two distinguishable particles, not covered in the present work.

${ }^{45}$ A qutrit is a unity of quantum information represented by a threestate system, in analogy with the qubit, embodied by a two-state fermion. 